\title{
miR-21 inhibitor suppresses cell proliferation and colony formation through regulating the PTEN/AKT pathway and improves paclitaxel sensitivity in cervical cancer cells
}

\author{
GUOHUI DU, DONGMEI CAO and LINGZHENG MENG
}

Department of Pharmacy, Tangshan Maternity and Child Health Care Hospital, Tangshan, Hebei 063000, P.R. China

Received October 30, 2015; Accepted November 18, 2016

DOI: $10.3892 / \mathrm{mmr} .2017 .6340$

\begin{abstract}
The present study aimed to investigate the role and the molecular mechanisms underlying the effects of microRNA-21 (miR-21) on the proliferation, apoptosis and colony formation of cervical cancer cells, and to examine the role of miR-21 in mediating the sensitivity of cervical cancer cells to paclitaxel (PTX). Reverse transcription-quantitative polymerase chain reaction was employed to determine the level of miR-21 in various cervical cancer and normal cervical cells. The results revealed that the expression levels of miR-21 in cervical cancer cells were markedly higher when compared with normal cervical cells. Subsequently, a miR-21 inhibitor or negative control (NC) was transfected into cervical cancer cells. Cell viability, colony formation and apoptosis were then analyzed using an MTT assay, crystal violet and Annexin V-fluorescein isothiocyanate/propidium iodide staining, respectively. The protein expression level of B-cell lymphoma-2 (Bcl-2), Bcl-2-associated X (Bax), programmed cell death 4 (PDCD4), survivin, c-myc, phosphatase and tensin homolog (PTEN) and phosphorylated (p)-AKT were determined by western blot analysis. The sensitivity of cervical cancer cells to PTX $(25,50$ and $100 \mu \mathrm{g} / \mathrm{ml})$ was characterized using an MTT assay. The results demonstrated that the miR-21 inhibitor promoted apoptosis of cervical cancer cells and suppressed their proliferation and colony formation when compared with the NC. In addition, the expression levels of Bcl-2, survivin, c-myc and p-AKT were significantly downregulated in cells transfected with the miR-21 inhibitor, whilst the expression levels of Bax, PDCD4 and PTEN were significantly upregulated. Furthermore, the miR-21 inhibitor significantly enhanced the inhibition efficacy of PTX at a range of concentrations in cervical cancer cells. It was concluded
\end{abstract}

Correspondence to: Professor Dongmei Cao, Department of Pharmacy, Tangshan Maternity and Child Health Care Hospital, 14 Jianshe Road, Tangshan, Hebei 063000, P.R. China

E-mail: caodm1011@sina.com

Key words: microRNA-21, cervical cancer, cell proliferation, colony formation, phosphatase and tensin homolog/AKT signaling pathway, paclitaxel that inhibition of miR-21 suppressed cell proliferation and colony formation through regulating the PTEN/AKT pathway, and improved PTX sensitivity in cervical cancer cells. The results of the present study may contribute to the development of miRNA-based cervical cancer therapy in the future.

\section{Introduction}

Cervical cancer is a common gynecological malignant disease with the second highest morbidity and the third-highest mortality rates among female patients with malignant tumors (1). Chemotherapy combined with surgery is a common method of treatment, with paclitaxel (PTX) as the primary chemotherapeutic agent used to treat patients with cervical cancer. However, a side effect of PTX is severe toxicity, and drug resistance may occur. Therefore, increasing PTX sensitivity and efficacy is an important aim for the treatment of patients with cervical cancer.

The age of onset of cervical cancer is becoming increasingly younger $(2,3)$. The prognosis of patients in the advanced stages of the disease is very poor, with a low five-year survival rate $(2,3)$. Thus, the early diagnosis of cervical cancer based on specific high-performance biomarkers may be an effective approach to reduce morbidity and mortality and improve prognosis. MicroRNAs (miRNAs) are endogenous non-coding RNAs with highly-conserved sequences. miRNAs bind to the 3'-untranslated region of target mRNA sequences and subsequently repress their translation and expression (4). In addition, miRNAs are closely correlated with the development and progression of cervical cancer $(5,6)$. They serve important roles in regulating the proliferation, apoptosis, invasion and metastasis of cervical cancer cells. Previous studies have demonstrated that miRNAs such as miR-21, miR-218, miR-10a, miR-196a, miR-132, and miR-148a increase the sensitivity of cervical cancer cells to chemotherapeutic agents, such as PTX (5-8). Therefore, miRNAs may provide an alternative strategy for the early diagnosis and prognosis of cervical cancer $(5,6)$.

miR-21 is the only miRNA that is highly expressed in 540 samples of human solid tumors including those of the lung, breast, stomach, prostate, colon and pancreas (9). It has been confirmed to function as an oncogene during cancer pathogenesis (9). Zeng et al (5) demonstrated that miR-21 was overexpressed in cervical intraepithelial neoplasia tissues 
and in cervical cancer tissues through reverse transcription-quantitative polymerase chain reaction (RT-qPCR) assay analysis, which was $\sim 6$-fold higher when compared to normal tissues. Deftereos et al (10) reported that miR-21 was highly expressed in invasive cervical carcinomas when compared with cervical intraepithelial neoplasia tissues. Lui et al (11) determined the expression of 166 miRNAs in normal cervical cells and cervical cancer cells, which indicated that miR-21 demonstrated the most significant upregulation in cervical cancer cells. These results indicate that miR-21 may be extensively involved in the development and progression of cervical cancer. In the current study, the effects of silenced miR-21 expression on the proliferation, colony formation and apoptosis of cervical cancer cells were explored. Furthermore, the sensitivity of cervical cancer cells to PTX following forced miR-21 downregulation was investigated.

\section{Materials and methods}

Materials and cell culture. The Annexin V-fluorescein isothiocyanate (FITC)/propidium iodide (PI) apoptosis detection kit, Hoechst 33258 staining kit, radioimmunoprecipitation assay buffer, enhanced chemiluminescence solution, and the bicinchoninic acid (BCA) assay kit were purchased from Beyotime Institute of Biotechnology (Haimen, China). Lipofectamine $^{\mathrm{TM}}$ 2000, TRIzol reagent, and the SuperScript III One-Step RT-PCR kit were purchased from Invitrogen; Thermo Fisher Scientific, Inc. (Waltham, MA, USA). MTT reagent and trypsin were purchased from Gibco; Thermo Fisher Scientific, Inc. PTX (lot no. 10382-201102; purity 99.6\%) was purchased from the National Institute for the Control of Pharmaceutical and Biological Products (Beijing, China). Rabbit anti-B-cell lymphoma-2 (Bcl-2), anti-Bcl-2-associated $\mathrm{X}$ (Bax), anti-survivin, and anti-c-myc monoclonal antibodies were obtained from Epitomics (cat. nos. 1017-1, 1063-1, 2463-1 and 1472-1, respectively; Burlingame, CA, USA). Rabbit anti-programmed cell death 4 (PDCD4), anti-phosphate and phosphatase and tensin homolog (PTEN), anti-Ser/Thr protein kinase (AKT) and anti-phosphorylated (p)-AKT monoclonal antibodies were purchased from Cell Signaling Technology, Inc. (cat. nos. 9535, 5384, 4685, and 12178, respectively, Danvers, MA, USA).

Cervical cell lines, C-33A, CaSki, SiHa, HeLa and ME-180 (cat. nos. TCHu176, TCHu137, TCHu113, TCHu187 and TCHu180, respectively) were obtained from The Cell Bank of Type Culture Collection of the Chinese Academy of Sciences (Shanghai, China). The normal cervical epithelial squamous cell line H8 was purchased from the Institute of Basic Medical Sciences of the Chinese Academy of Medical Sciences, (Beijing, China). Cells were cultured in Dulbecco's Modified Eagle's medium (Hyclone; GE Healthcare Life Sciences, Logan, UT, USA) supplemented with $10 \%$ (v/v) fetal bovine serum (Hyclone; GE Healthcare Life Sciences).

Preparation of miRNA. miR-21 antisense oligonucleotide (asOGC); (5'-UCA ACAUCAGUCUGAUAAGCU-3') and the negative control (NC; 5'-GAUGUUGAAACAUCA GUCUGA-3') were synthesized by Shanghai GenePharma Co., Ltd., (Shanghai, China). The quantity of miRNA and Lipofectamine 2000 used for gene transfection was established according to the manufacturer's instructions (Invitrogen; Thermo Fisher Scientific, Inc.). HeLa cells were seeded in 96-well plates at a density of $5 \times 10^{3}$ cells/well. When cells reached $50 \%$ confluence, they were transfected with miR-21 asOGC or NC using Lipofectamine ${ }^{\mathrm{TM}}$ 2000. Cells were harvested for analysis at $48 \mathrm{~h}$ following transfection.

miR-21 levels in normal cervical cells and cervical cancer cells. Total RNA from normal and cancer cells was extracted using TRIzol reagent according to the manufacturer's instructions (Invitrogen; Thermo Fisher Scientific, Inc.) in an RNase-free environment. RNA was reverse transcribed into cDNA and subsequently amplified by PCR using the One-Step RT-PCR kit (Invitrogen; Thermo Fisher Scientific, Inc.). Primers (20 $\mu \mathrm{M}$; Sangon Biotechnology Co., Ltd., Shanghai, China; Table I) were added into the PCR reaction mixture (total volume, $25 \mu \mathrm{l}$ ). The thermal cycling parameters included 35 cycles of denaturation for $45 \mathrm{sec}$ at $94^{\circ} \mathrm{C}$, annealing for $45 \mathrm{sec}$ at $59^{\circ} \mathrm{C}$ and elongation for $60 \mathrm{sec}$ at $72^{\circ} \mathrm{C}$. The PCR product (5 $\mu \mathrm{l}$ each lane) was separated by gel electrophoresis on a $2 \%$ $(w / v)$ agarose gel. Electrophoresis strips were examined with Quantity One software v.4.6.2 (Bio-Rad Laboratories, Inc., Hercules, CA, USA) using a gel imaging system (ChemiDoc ${ }^{\mathrm{TM}}$ XRS; Bio-Rad Laboratories, Inc.).

Cell viability. At $48 \mathrm{~h}$ following transfection, $20 \mu \mathrm{l}$ MTT $(5 \mathrm{mg} / \mathrm{ml})$ was added to each well, and cells were cultured for a further $4 \mathrm{~h}$. The culture media was then discarded and $150 \mu \mathrm{l}$ dimethyl sulfoxide was added to each well. Plates were agitated to thoroughly dissolve the crystals, before the absorbance was read at $560 \mathrm{~nm}$ using a microplate reader (Infinite M200; Tecan Trading AG, Zurich, Switzerland). The relative cell viability was calculated by comparing the absorbance values of treated cells with the untreated control cells.

Colony formation. At $48 \mathrm{~h}$ following transfection of HeLa cells with miR-21 asOGC or NC, cells were fixed with $10 \%(\mathrm{w} / \mathrm{v})$ formaldehyde and stained with $0.1 \%(\mathrm{w} / \mathrm{v})$ crystal violet for $30 \mathrm{~min}$ at room temperature. The staining solution was then discarded carefully and each well was washed with water. The plates were then inverted on absorbent paper to dry. Finally, the cells were visualized in five fields under a fluorescence microscope (AF6000, Leica Microsystems GmbH, Wetzlar, Germany). Results were expressed as the average number of cells in every visual field.

Cell apoptosis with Annexin V-FITC/PI staining. The Annexin V-FITC/PI apoptosis detection kit was utilized to evaluate cell apoptosis according to the manufacturer's instructions (Beyotime Institute of Biotechnology). At $48 \mathrm{~h}$ following transfection of HeLa cells with miR-21 asOGC and NCs, cells were digested with $0.25 \%$ (w/v) trypsin (without EDTA), washed with phosphate-buffered saline (PBS), and collected by centrifugation at $800 \times \mathrm{g}$ for $5 \mathrm{~min}$ at room temperature. Cells were the resuspended in $500 \mu \mathrm{l}$ binding buffer, $5 \mu \mathrm{l}$ Annexin V-FITC stain and $5 \mu \mathrm{l}$ PI, before they were incubated for $10 \mathrm{~min}$ at room temperature in the dark. Cell apoptosis was evaluated using a flow cytometer with CellQuest Pro v.5.2 software (BD FACScan; BD Biosciences, Franklin Lakes, NJ, USA). 
Cell apoptosis with Hoechst 33258 staining. The Hoechst 33258 kit was employed to evaluate cell apoptosis according to the manufacturer's instructions (Beyotime Institute of Biotechnology). At $48 \mathrm{~h}$ following transfection, cells were fixed with $4 \%(\mathrm{w} / \mathrm{v})$ paraformaldehyde for $15 \mathrm{~min}$ at room temperature, then washed three times with $\mathrm{PBS}$, before they were incubated with Hoechst $33258(10 \mu \mathrm{g} / \mathrm{ml})$ for $15 \mathrm{~min}$ at room temperature in the dark. Finally, cells were washed three times with PBS and observed in five fields of view under a fluorescence microscope. Results were expressed as the average number of cells in every visual field.

Western blot analysis. At $48 \mathrm{~h}$ following transfection, cells were collected and lysed in radioimmunoprecipitation assay buffer. Lysates were agitated using a vortex for $30 \mathrm{sec}$ every $10 \mathrm{~min}$. Following $40 \mathrm{~min}$, the supernatant was carefully separated from the mixture by centrifugation at 7,000 $\mathrm{x} g$ for $10 \mathrm{~min}$ at $4^{\circ} \mathrm{C}$ to obtain total protein. The protein concentration was determined using a BCA kit. Proteins were loaded (20 $\mu \mathrm{g}$ per lane) to perform SDS-PAGE (10\% separation gel, 5\% concentrated gel) electrophoresis and transferred to a polyvinylidene difluoride membrane using a wet transfer methodology. Membranes were blocked with $5 \%(\mathrm{w} / \mathrm{v})$ non-fat milk buffer at room temperature for $1 \mathrm{~h}$, then incubated with primary antibodies (dilution 1:100) overnight at $4^{\circ} \mathrm{C}$, and then rinsed with PBS and incubated with a secondary antibody buffer (dilution 1:100) for $2 \mathrm{~h}$ at room temperature. Following a further rinse with PBS, enhanced chemiluminescence solution was added to the membrane, which was then exposed using a gel imaging system (ChemiDoc $^{\mathrm{TM}}$ XRS; Bio-Rad Laboratories, Inc.). Quantity One software v4.6.2 (Bio-Rad Laboratories, Inc.) was employed to determine the gray values of proteins. GADPH served as the internal control.

PTX sensitivity. At $48 \mathrm{~h}$ following transfection, cells were trypsinized to produce single-cell suspensions and seeded in 96 -well plates at a density of $5 \times 10^{3}$ cells/well. After $24 \mathrm{~h}$, cells were incubated with 25, 50, and $100 \mu \mathrm{g} / \mathrm{ml}$ PTX for a further $48 \mathrm{~h}$. Cell viability was evaluated with an MTT assay using the aforementioned procedures.

Statistical analysis. Data are expressed as the mean \pm standard deviation $(n=6)$. Statistical analysis was performed using a Student's t-test with SPSS software (version 17.0; SPSS, Inc., Chicago, IL, USA). P $<0.05$ was considered to indicate a statistically significant difference.

\section{Results}

miR-21 expression levels in normal cervical cells and cervical cancer cells. miR-21 levels in normal cervical cells and cervical cancer cells were evaluated by RT-qPCR analysis and the results are shown in Fig. 1. miR-21 levels in cervical cancer cell lines HeLa, CaSki, SiHa, C-33A and ME-180 were 2.8-4.0-fold higher when compared with the normal cervical cell line, H8 ( $\mathrm{P}<0.01$ for each comparison; Fig. 1). Among the five cervical cancer cell lines, HeLa cells exhibited the highest expression levels of miR-21 (Fig. 1).

Effect of miR-21 asOGC on the viability of cervical cancer cells. The viability of HeLa cells transfected with the miR-21
Table I. Primers used for reverse transcription-quantitative polymerase chain reaction analysis.

\begin{tabular}{ll}
\hline Gene & \multicolumn{1}{c}{ Primer $\left(5^{\prime}-3^{\prime}\right)$} \\
\hline miR-21 & F: GCCGCTAGCTTATCAGACTGATGT \\
& R: GTGCAGGGTCCGAGGT \\
GADPH & F: AGCCACATCGCTCAGACA \\
& R: TGGACTCCACGACGTACT \\
\hline
\end{tabular}

miR-21, microRNA-21; F, forward; R, reverse.

Table II. Viability of HeLa cells transfected with the miR-21 asOGC or the miR-21 NC.

\begin{tabular}{ll}
\hline Group & Cell viability \\
\hline miR-21 NC & $0.68 \pm 0.04$ \\
miR-21 asOGC & $0.40 \pm 0.01^{\text {a }}$ \\
\hline
\end{tabular}

${ }^{\mathrm{a}} \mathrm{P}<0.01$ vs. miR-21 NC.

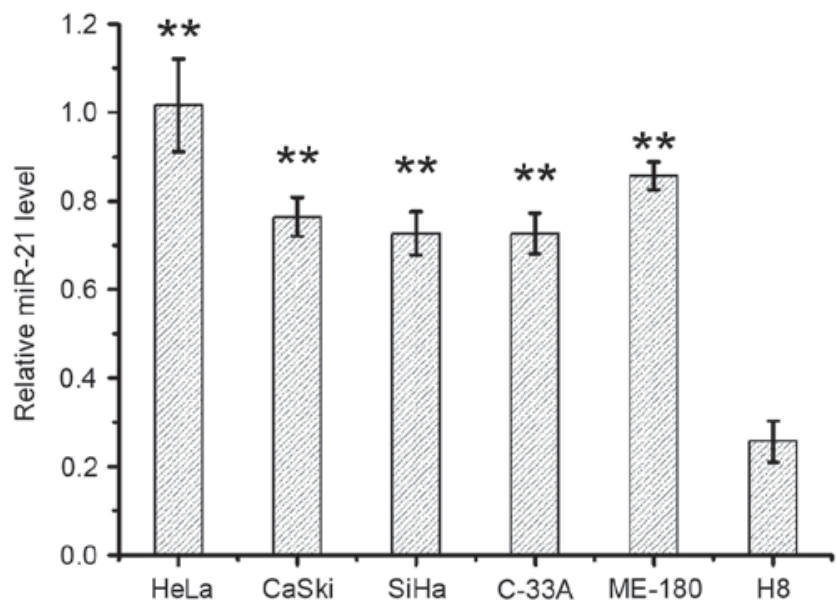

Figure 1. miR-21 expression levels in cervical cancer cells (HeLa, CaSki, SiHa, C-33A, and ME-180) and normal cervical cells (H8) as determined by reverse transcription-quantitative polymerase chain reaction. ${ }^{* *} \mathrm{P}<0.01 \mathrm{vs}$. H8 cells. miR-21, microRNA-21.

asOGC or the miR-21 NC was determined by MTT assay. As demonstrated in Table II, compared with the NC, miR-21 asOGC transfection reduced the proliferation of HeLa cells $(\mathrm{P}=0.007)$.

Effect of miR-21 asOGC on the colony formation of cervical cancer cells. As demonstrated in Table III, miR-21 asOGC-transfected HeLa cells exhibited a significant reduction in colony number when compared with NC-transfected cells $(\mathrm{P}=0.002)$.

Effect of an miR-21 asOGC on the apoptosis of cervical cancer cells. The number of apoptotic HeLa cells transfected with miR-21 asOGC or NC was evaluated by Annexin 
Table III. Colony formation in HeLa cells transfected with the miR-21 asOGC or the miR-21 NC.

\begin{tabular}{lc}
\hline Group & Cell colony number \\
\hline miR-21 NC & $152.4 \pm 114.7$ \\
miR-21 asOGC & $58.4 \pm 5.7^{\mathrm{a}}$ \\
\hline
\end{tabular}

${ }^{\mathrm{a}} \mathrm{P}<0.01$ vs. $\mathrm{miR}-21 \mathrm{NC}$.

V-FITC/PI staining (Fig. 2A) and Hoechst 33258 staining (Fig. 2B). Following transfection of cells with the miR-21 asOGC, the late apoptosis ratio of HeLa cells was significantly enhanced when compared with the $\mathrm{NC}$ group $(\mathrm{P}=0.001$; Fig. 2A). Similarly, Hoechst 33258 staining revealed significantly more apoptotic HeLa cells with the miR-21 asOGC (38.89 $\pm 3.22 \%$; Fig. 2B) compared with the NC $(5.29 \pm 0.42 \%$; $\mathrm{P}=0.001$; Fig. 2B). The results of the present study, therefore, suggest that suppression of miR-21 expression induces apoptosis in cervical cancer cells.

Effect of miR-21 asOGC on the expression of PDCD4, Bax, Bcl-2, survivin, c-myc and PTEN/AKT in cervical cancer cells. The expression of signaling molecules in HeLa cells transfected with an miR-21 asOGC or a NC was assessed by western blot analysis, as demonstrated in Fig. 3. As an internal control, similar GADPH protein band densities were observed between the two groups, thus confirming the reliable evaluation of the western blotting results.

Notably, a significant increase in the protein expression levels of PDCD4 and Bax were observed in HeLa cells transfected with the miR-21 asOGC when compared with cells transfected with the $\mathrm{NC}(\mathrm{P}=0.003$ and $\mathrm{P}=0.002$, respectively; Fig. 3A). Conversely, a significant reduction in Bcl-2 expression was observed in cells transfected with the miR-21 asOGC when compared with the $\mathrm{NC}$ group ( $\mathrm{P}=0.002$; Fig. $3 \mathrm{~A})$.

Compared with the NC group, HeLa cells expressed significantly lower levels of survivin and c-myc following transfection with the miR-21 asOGC $(\mathrm{P}=0.004$ and $\mathrm{P}=0.003$, respectively; Fig. $3 \mathrm{~B}$ ), which demonstrated that the miR-21 asOGC downregulated the expression of survivin and c-myc in cervical cancer cells. Similar levels of AKT protein expression were observed in the miR-21 asOGC-transfected HeLa cells and the NC-transfected cells (Fig. 3C).

However, significantly increased levels of PTEN and decreased levels of p-AKT were detected in HeLa cells transfected with the miR-21 asOGC when compared with those transfected with the $\mathrm{NC}(\mathrm{P}=0.002$ and $\mathrm{P}=0.004$, respectively; Fig. 3C). Therefore, transfection of an miR-21 asOGC into cervical cancer cells increased the levels of PTEN and decreased the phosphorylation of AKT.

Effect of an miR-21 inhibitor on the sensitivity of cervical cancer cells to PTX. The sensitivity of HeLa cells to PTX was evaluated using an MTT assay and the results are presented in Fig. 4. Compared with the NC group, transfection of cells with the miR-21 asOGC significantly reduced the viability of HeLa cells following incubation with 25,50 and $100 \mu \mathrm{g} / \mathrm{ml}$

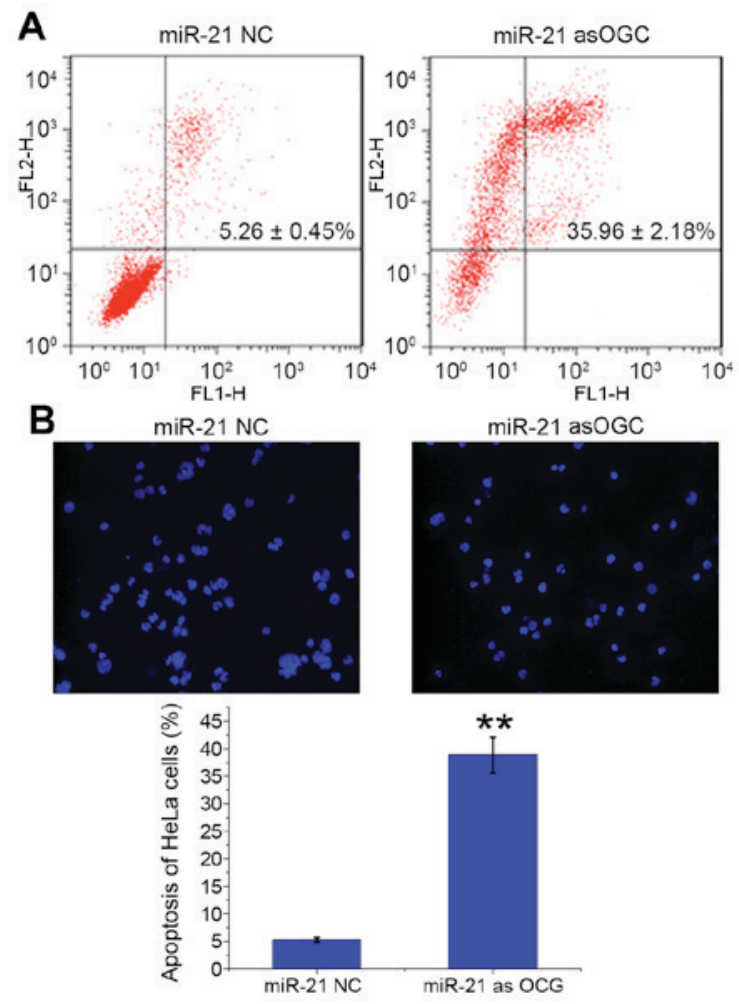

Figure 2. Apoptosis of HeLa cells transfected with miR-21 antisense oligonucleotide or NC, which was assessed by (A) Annexin V fluorescein isothiocyanate/propidium iodide staining and (B) Hoechst 33258 staining. ${ }^{* * *} \mathrm{P}<0.01$ vs. miR-21 NC. miR-21, microRNA-21; NC, negative control; FITC, fluorescein isothiocyanate; PI, propidium iodide.

PTX by $54-63 \%(\mathrm{P}=0.005, \mathrm{P}=0.006$ and $\mathrm{P}=0.007$, respectively; Fig. 4), which suggests that the miR-21 asOGC was capable of enhancing the sensitivity of cervical cancer cells to PTX.

\section{Discussion}

The human papillomavirus (HPV) is one of the most important causes of cervical cancer, and chromosome 17q23.3 is the most common integration site for the viral genome $(7,12)$. The subsequent genetic and epigenetic alterations exert significant effects on tumorigenesis involving miRNA sequences localized near the integration site $(7,12)$. A previous study demonstrated that miR-21, located in the FRA17B fragile region of 17q23.3, exhibits the highest expression levels in HPV16-positive cervical cancer tissues (12). In addition, it was demonstrated to be overexpressed in cervical cancers when compared with normal cervical tissues $(5,10,11)$. Furthermore, investigations involving miRNA chip screening of 363 tumor samples, including breast cancer, colon cancer, lung cancer, pancreatic cancer, prostate cancer and gastric cancer, as well as 177 corresponding normal tissue samples, demonstrated that miR-21 was the only miRNA with upregulated expression in all tumor samples (9). These results suggested that miR-21 was overexpressed in cervical cancer, as well as a number of additional cancer tissues. Therefore, miR-21 may be considered to be an oncogenic miRNA sequence. In the present study, miR-21 was demonstrated to be overexpressed in various cervical cancer cells when compared with normal 
A
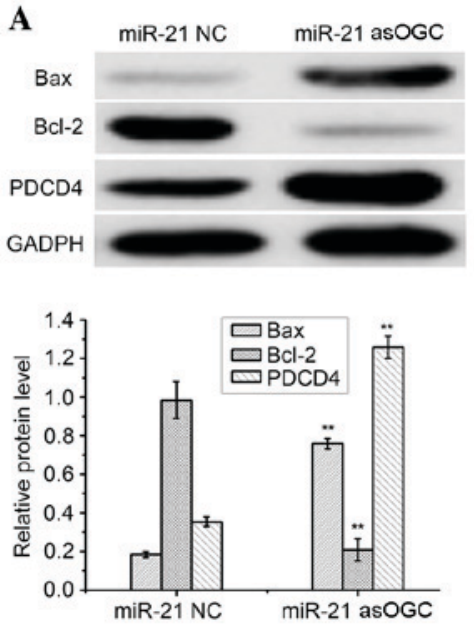

B
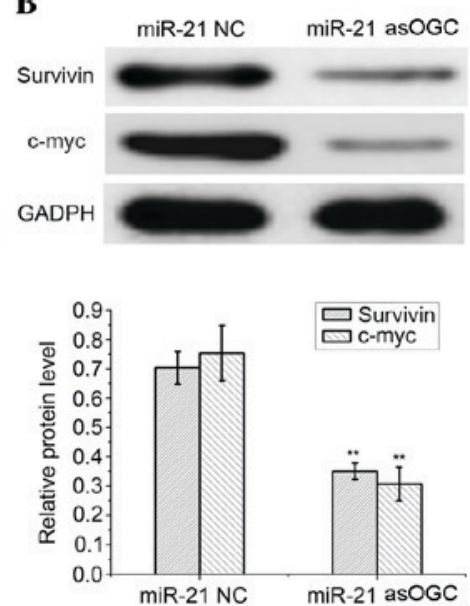
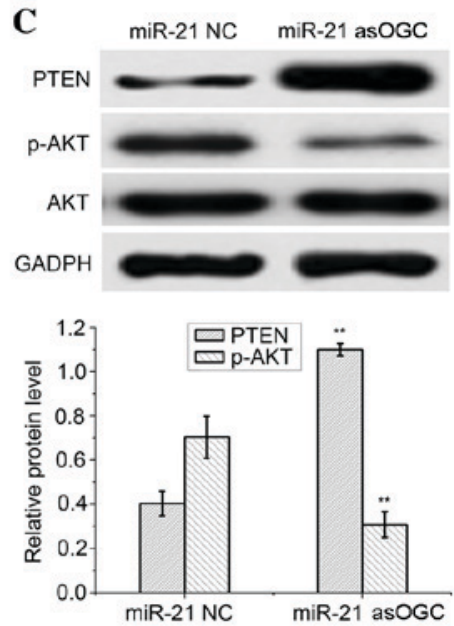

Figure 3. Qualitative and quantitative western blot analysis of (A) Bax, Bcl-2, PDCD4, (B) survivin, c-myc and (C) PTEN, AKT and p-AKT in HeLa cells transfected with a miR-21 antisense oligonucleotide or NC. ${ }^{* *} \mathrm{P}<0.01$ vs. miR-21 NC. Bax, Bcl-2-associated X; Bcl-2, B-cell lymphoma 2; PDCD4, programmed cell death protein 4; PTEN, phosphatase and tensin homolog; p-AKT, phosphorylated AKT; miR-21, microRNA-21; NC, negative control.

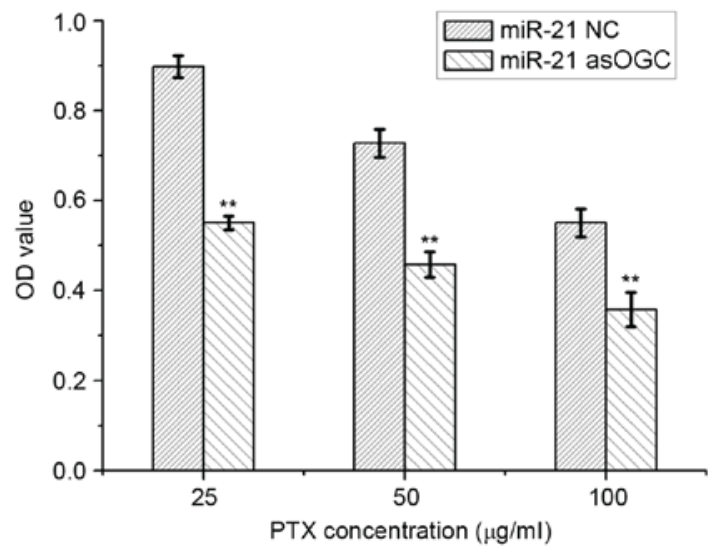

Figure 4. The sensitivity of HeLa cells transfected with miR-21 NC and a miR-21 antisense oligonucleotide to different concentrations of PTX, which was evaluated using an MTT assay. ${ }^{* *} \mathrm{P}<0.01$ vs. miR-21 NC. miR-21, microRNA-21; NC, negative control; PTX, paclitaxel; OD, optical density.

cervical cells, which was consistent with the findings of a previous report (11). These results provided additional evidence to suggest that miR-21 may be oncogenic in cervical cancer, and may present a novel target for the diagnosis and treatment of patients with cervical cancer. In addition, as the highest expression of miR-21 was observed in the HeLa cell line, these cells were therefore selected as a model cervical cancer cell line for the subsequent investigations.

In the present study, an miR-21 asOGC was applied as an miR-21 inhibitor to downregulate intracellular miR-21 levels in cervical cancer cells. It was demonstrated that transfection of the miR-21 asOGC suppressed the proliferation and colony formation, and enhanced apoptosis of HeLa cells. This result was in accordance with previous reports, where an miR-21 inhibitor was observed to suppress the proliferation and promote apoptosis of cervical, pancreatic, breast and prostate cancer cells, as well as tongue squamous carcinoma cells (13-15).

The balance between cell proliferation and apoptosis strongly influences tumor development and progression $(16,17)$.
If cell apoptosis is inhibited, cell hyperplasia may occur. Mutant cells that are unable to undergo apoptosis, may proliferate uncontrollably, leading to tumorigenesis $(16,17)$. Therefore, the inhibition of cell proliferation and induction of cell apoptosis may be an effective strategy for cancer therapy. Cell proliferation and apoptosis are regulated by related genes, such as the Bcl-2 family, which has been extensively investigated $(16,17)$. $\mathrm{Bcl}-2$ promotes the proliferation of cancer cells $(16,17)$. In addition, Bcl-2 is overexpressed in cervical cancer cells and is closely correlated with the development and progression of cervical cancer (18). Bax belongs to the Bcl-2 family and forms dimers with Bcl-2 to regulate the balance between cell proliferation and apoptosis. Bax expression is downregulated, or even completely absent in cervical cancer cells, and its gene polymorphism [BAX-248G $>$ A (rs4645878)] is intensively correlated with the progression of cervical cancer (19). Therefore, the upregulation of Bax expression and downregulation of Bcl-2 expression observed in miR-21 asOGC-transfected cells in the present study, may have been responsible for the increased apoptosis and reduction in cell proliferation of cervical cancer cells. A previous report revealed that altered regulation of miR-21 promoted cancer cell apoptosis by influencing diverse target genes associated with cell apoptosis, including Bcl-2, PDCD4 and PTEN (20). In the present study, a miR-21 asOGC elevated Bax expression and reduced Bcl-2 expression in HeLa cells. In previous studies, a downstream target gene of miR-21, PDCD4, was determined to be a tumor suppressor gene $(21,22)$. Its expression is absent in multiple cancers, including cervical cancer, and is closely associated with tumor development and progression (22). In addition, miR-21 has been demonstrated to promote the proliferation of cervical cancer cells via downregulation of PDCD4 expression (21). Therefore, suppression of miR-21 expression may inhibit the proliferation of HeLa cervical cancer cells (21), Colo206f colorectal cancer cells (23) and T89 G glioma cells (24), due to elevation of PDCD4 expression. The present study demonstrated that, following a reduction of intracellular miR-21 levels by transfection with a miR-21 asOGC, PDCD4 levels and cancer cell apoptosis was increased. 
Survivin, a regulator of cell mitosis, is a member of an apoptosis-suppressing protein family (25). To date, it is currently the most effective apoptosis-suppressing protein (25). The positive expression of survivin is correlated with clinical stage, lesion size, and degree of tumor differentiation (26). Overexpression of survivin has been demonstrated to elevate intracellular levels of the proto-oncogene c-myc, which subsequently accelerates tumorigenesis (26). Moreover, the expression of c-myc in cervical cancer tissues is notably higher when compared with that in normal cervical tissues, and c-myc is involved in the level of pathological differentiation and clinical stage of cancer tissues (27). Therefore, the decreased level of survivin and c-myc observed in miR-21 asOGC-transfected HeLa cells in the present study, may have led to inhibition of the proliferation of HeLa cells and may prevent tumor progression. The results of the current study demonstrated that miR-21 asOGC induced apoptosis and inhibited the proliferation of cervical cancer cells likely through the upregulation of Bax and PDCD4 and downregulation of Bcl-2, survivin and c-myc.

As a novel tumor suppressor gene, PTEN is a downstream target gene of miR-21, which is expressed at a low level, deleted, or mutated in a number of cancers (28). It dephosphorylates phosphatidyl inositol triphosphate into phosphatidyl inositol bisphosphate, and subsequently negatively regulates the phosphatidylinositol-3-kinase (PI3K)/AKT signaling pathway (29). Among the various signaling pathways that regulate tumor cell proliferation and apoptosis, the PI3K/AKT pathway is an anti-apoptotic pathway (29). This signaling pathway mediates cancer cell apoptosis, tumor angiogenesis, invasion and metastasis by regulating a variety of downstream target proteins. The positive rate of PTEN in cervical cancer tissues is significantly lower than that in normal cervical tissues, and its positive expression is associated with low clinical stage and reduced lymphatic metastasis (30). Numerous heterozygotes are deleted in cervical cancer tissues, which are located in D10S198 and D10S192 sites (31). It has been suggested that inactivation of the PTEN gene and inhibition of protein expression may promote the progression of cervical cancer $(32,33)$. However, the PI3K/AKT signaling pathway is abnormally activated in cervical cancer tissues (34). Tao et al (35) utilized oligonucleotides to inhibit the expression of miR-21 in HCT116 human colon cancer cells, and demonstrated that the proliferation and metastasis of these cells was suppressed by upregulation of PTEN expression. Therefore, overexpression of miR-21 in cervical cancer cells may inhibit the expression of PTEN and facilitate the proliferation and metastasis of cervical cancer cells (36). In the present study, when the expression of miR-21 was downregulated by an miR-21 asOGC in HeLa cells, the expression of PTEN was increased and the levels of p-AKT were significantly reduced. This may have been responsible for the observed increase in apoptosis and decrease in proliferation of miR-21 asOGC-transfected HeLa cells.

In addition, the miR-21 asOGC was capable of enhancing the sensitivity of HeLa cells to PTX at a series of increasing concentrations. This indicated that the miR-21 asOGC increased the therapeutic efficacy of PTX in vitro. PTX is a major chemotherapeutic agent for the treatment of cervical cancer, however it leads to severe side effects, including arrest of bone marrow function, anaphylactic reaction and damage to the liver and kidneys (37). Therefore, the dose of PTX is strictly controlled to alleviate the painful side effects, which may, however, compromise the efficacy (37). The results of the current study demonstrated that a miR-21 asOGC significantly enhanced the sensitivity of cervical cancer cells to PTX, and improved its efficacy, even at a low dose. Therefore, a miR-21 inhibitor could be applied as an adjuvant agent for the treatment of cervical cancer. However, the detailed mechanisms of action require further investigation.

In conclusion, miR-21 may serve an important role in the development and progression of cervical cancer. In the present study, cervical cancer cells exhibited high levels of miR-21 expression when compared with a normal cervical tissue cell line, which may underlie the aggressive biological behaviors of cervical tumors. Following the transfection of a miR-21 asOGC into cervical cancer cells, apoptosis of cervical cancer cells was increased, while their proliferation and colony formation was suppressed. This may have been through regulation of the PTEN/AKT signaling pathway, where Bcl-2, survivin and c-myc were negatively regulated, and Bax and PDCD4 were positively regulated. In addition, the miR-21 asOGC improved the sensitivity of cervical cancer cells to PTX. These findings may serve as guidelines for the development of miRNA-based agents and therapeutics for the treatment of cervical cancer.

\section{References}

1. Missaoui N, Hmissa S, Trabelsi A, Frappart L, Mokni M and Korbi S: Cervix cancer in Tunisia: Clinical and pathological study. Asian Pac J Cancer Prev 11: 235-238, 2010.

2. Feng Y, Cao T, Wang Y, Huang H, Xie Y and Liu J: Neoadjuvant chemotherapy followed by conization to spare fertility in cases of locally advanced cervical cancer: A case report and review of the literature. Mol Clin Oncol 5: 411-416, 2016.

3. Qin AQ, Liang ZG, Ye JX, Li J, Wang JL, Chen CX and Song HL: Significant efficacy of additional concurrent chemotherapy with radiotherapy for postoperative cervical cancer with risk factors: A systematic review and meta-analysis. Asian Pac J Cancer Prev 17: 3945-3951, 2016.

4. Gambari R, Brognara E, Spandidos DA and Fabbri E: Targeting oncomiRNAs and mimicking tumor suppressor miRNAs: New trends in the development of miRNA therapeutic strategies in oncology (Review). Int J Oncol 49: 5-32, 2016.

5. Zeng K, Zheng W, Mo X, Liu F, Li M, Liu Z, Zhang W and Hu X: Dysregulated microRNAs involved in the progression of cervical neoplasm. Arch Gynecol Obstet 292: 905-913, 2015.

6. Shen Y, Wang P, Li Y, Ye F, Wang F, Wan X, Cheng X, Lu W and $\mathrm{Xie} \mathrm{X}$ : miR-375 is upregulated in acquired paclitaxel resistance in cervical cancer. Br J Cancer 109: 92-99, 2013.

7. Pedroza-Torres A, López-Urrutia E, Garcia-Castillo V, Jacobo-Herrera N, Herrera LA, Peralta-Zaragoza O, López-Camarillo C, De Leon DC, Fernández-Retana J, Cerna-Cortés JF and Pérez-Plasencia C: MicroRNAs in cervical cancer: Evidences for a miRNA profile deregulated by HPV and its impact on radio-resistance. Molecules 19: 6263-6281, 2014.

8. Liu S, Cheng X, Zheng H and Xie R: Clinical efficacy and safety of paclitaxel plus cisplatin neoadjuvant treatment on locally advanced cervical cancer. Zhongguo Linchuang Yaolixue Zazhi 31: 432-434, 2015 (In Chinese).

9. Volinia S, Calin GA, Liu CG, Ambs S, Cimmino A, Petrocca F, Visone R, Iorio M, Roldo C, Ferracin M, et al: A microRNA expression signature of human solid tumors defines cancer gene targets. Proc Natl Acad Sci USA 103: 2257-2261, 2006.

10. Deftereos G, Corrie SR, Feng Q, Morihara J, Stern J, Hawes SE and Kiviat NB: Expression of mir-21 and mir-143 in cervical specimens ranging from histologically normal through to invasive cervical cancer. PLoS One 6: e28423, 2011. 
11. Lui WO, Pourmand N, Patterson BK and Fire A: Patterns of known and novel small RNAs in human cervical cancer. Cancer Res 67: 6031-6043, 2007

12. Wang X, Tang S, Le SY, Lu R, Rader JS, Meyers C and Zheng ZM: Aberrant expression of oncogenic and tumor-suppressive microRNAs in cervical cancer is required for cancer cell growth. PLoS One 3: e2557, 2008.

13. Wang XM, Xu J, Cheng ZQ, Peng QZ, Hu JT, Gao LK, Zhang SF and Jin HT: Study on effects of microRNA-21 antisense oligonucleotide in vivo and in vitro on bionomics of human cervical squamous carcinoma cell lines SiHa. Zhonghua Bing Li Xue Za Zhi 41: 254-259, 2012 (In Chinese).

14. Zhu W and Xu B: MicroRNA-21 identified as predictor of cancer outcome: A meta-analysis. PLoS One 9: e103373, 2014.

15. Wang Y,Zhu Y,Lv P and Li L: Targeting miR-21 with AS-miR-21 suppresses aggressive growth of human tongue squamous cell carcinoma in vivo. Int J Clin Exp Pathol 8: 4773-4781, 2015.

16. Ouyang L, Shi Z, Zhao S, Wang FT, Zhou TT, Liu B and Bao JK: Programmed cell death pathways in cancer: A review of apoptosis, autophagy and programmed necrosis. Cell Prolif 45: 487-498, 2012

17. Zeestraten EC, Benard A, Reimers MS, Schouten PC, Liefers GJ, van de Velde CJ and Kuppen PJ: The prognostic value of the apoptosis pathway in colorectal cancer: A review of the literature on biomarkers identified by immunohistochemistry. Biomark Cancer 5: 13-29, 2013

18. Zhang Y, Yang H, Barnie PA, Yang P, Su Z, Chen J, Jiao Z, Lu L, Wang $\mathrm{S}$ and $\mathrm{Xu} \mathrm{H}$ : The expression of Toll-like receptor 8 and its relationship with VEGF and Bcl-2 in cervical cancer. Int J Med Sci 11: 608-613, 2014.

19. Fernandes AT, Rocha NP, Vendrame E, Russomano F, Grinsztejn BJ, Friedman RK, Pinto AC, Klumb EM, Avvad E, Macedo J, et al: Polymorphism in apoptotic BAX (-248G>A) gene but not in anti-apoptotic BCL2 $(-938 \mathrm{C}>\mathrm{A})$ gene and its protein and mRNA expression are associated with cervical intraepithelial neoplasia. Apoptosis 20: 1347-1357, 2015.

20. Huang Y, He Y and Li J: MicroRNA-21: A central regulator of fibrotic diseases via various targets. Curr Pharm Des 21: 2236-2242, 2015.

21. Yao Q, Xu H, Zhang QQ, Zhou H and Qu LH: MicroRNA-21 promotes cell proliferation and down-regulates the expression of programmed cell death 4 (PDCD4) in HeLa cervical carcinoma cells. Biochem Biophys Res Commun 388: 539-542, 2009.

22. Lankat-Buttgereit B and Göke R: The tumour suppressor Pded4 Recent advances in the elucidation of function and regulation. Biol Cell 101: 309-317, 2009.

23. Asangani IA, Rasheed SA, Nikolova DA, Leupold JH, Colburn NH, Post S and Allgayer H: MicroRNA-21 (miR-21) post-transcriptionally downregulates tumor suppressor Pded4 and stimulates invasion, intravasation and metastasis in colorecta cancer. Oncogene 27: 2128-2136, 2008.
24. Chen Y, Liu W, Chao T, Zhang Y, Yan X, Gong Y, Qiang B, Yuan J, Sun M and Peng X: MicroRNA-21 down-regulates the expression of tumor suppressor PDCD4 in human glioblastoma cell T98G. Cancer Lett 272: 197-205, 2008.

25. Roy K, Singh N, Kanwar RK and Kanwar JR: Survivin modulators: An updated patent review (2011-2015). Recent Pat Anticancer Drug Discov 11: 152-169, 2016.

26. Lu D, Yin X and Xiao Q: Expressions of PTEN and Survivin in the progression of cervical neoplasia and their clinical significances. Zhongguo Fuyou Baojian 28: 4721-4724, 2013 (In Chinese).

27. Hu Q and Liu K: The expression of CIP2A and c-Myc and their correlation analysis in cervical carcinoma tissues. Zhong Qing Yi Xue Bian Ji Bu 44: 1072-1074, 2015 (In Chinese).

28. Wang Y and Dai B: PTEN genomic deletion defines favorable prognostic biomarkers in localized prostate cancer: A systematic review and meta-analysis. Int J Clin Exp Med 8: 5430-5437, 2015.

29. Wang LL, Hao S, Zhang S, Guo LJ, Hu CY, Zhang G, Gao B, Zhao JJ, Jiang Y, Tian WG, et al: PTEN/PI3K/AKT protein expression is related to clinicopathologic features and prognosis in breast cancer with axillary lymph node metastases. Hum Pathol pii: S0046-8177, 2016.

30. Bu L, Ma Y and Shi S : Expressions of CD31, CD105 and PTEN in cervical cancer and the clinical pathological significance. Zhongguo Fuyou Baojian 30: 1446-1449, 2015.

31. Rizvi MM, Alam MS, Mehdi SJ, Ali A and Batra S: Allelic loss of 10q23.3, the PTEN gene locus in cervical carcinoma from Northern Indian population. Pathol Oncol Res 18: 309-313, 2012.

32. Qi Q, Ling Y, Zhu M, Zhou L, Wan M, Bao Y and Liu Y: Promoter region methylation and loss of protein expression of PTEN and significance in cervical cancer. Biomed Rep 2: 653-658, 2014

33. Lu D, Qian J, Yin X, Xiao Q, Wang C and Zeng Y: Expression of PTEN and survivin in cervical cancer: Promising biological markers for early diagnosis and prognostic evaluation. Br J Biomed Sci 69: 143-146, 2012.

34. Schwarz JK, Payton JE, Rashmi R, Xiang T, Jia Y, Huettner P, Rogers BE, Yang Q, Watson M, Rader JS and Grigsby PW: Pathway-specific analysis of gene expression data identifies the PI3K/Akt pathway as a novel therapeutic target in cervical cancer. Clin Cancer Res 18: 1464-1471, 2012.

35. Tao YJ, Li YJ, Zheng W, Zhao JJ, Guo MM, Zhou Y, Qin NL, Zheng $\mathrm{J}$ and $\mathrm{Xu} \mathrm{L}$ : Antisense oligonucleotides against microRNA-21 reduced the proliferation and migration of human colon carcinoma cells. Cancer Cell Int 15: 77, 2015.

36. Xu J, Zhang W, Lv Q and Zhu D: Overexpression of miR-21 promotes the proliferation and migration of cervical cancer cells via the inhibition of PTEN. Oncol Rep 33: 3108-3116, 2015.

37. Eskander RN and Tewari KS: Chemotherapy in the treatment of metastatic, persistent, and recurrent cervical cancer. Curr Opin Obstet Gynecol 26: 314-321, 2014. 